\title{
Complete Genome and Plasmid Sequence Data of Three Strains of Xanthomonas arboricola pv. corylina, the Bacterium Responsible for Bacterial Blight of Hazelnut
}

\author{
Joël F. Pothier, ${ }^{1, \dagger}$ Monika Kałużna, ${ }^{2}$ Andjelka Prokić, ${ }^{3}$ Aleksa Obradović, ${ }^{3}$ and Fabio Rezzonico ${ }^{1}$ \\ ${ }^{1}$ Environmental Genomics and Systems Biology Research Group, Institute for Natural Resource Sciences, \\ Zurich University of Applied Sciences (ZHAW), Wädenswil, Switzerland \\ ${ }^{2}$ The National Institute of Horticultural Research, Konstytucji 3 Maja 1/3, 96-100 Skierniewice, Poland \\ ${ }^{3}$ University of Belgrade, Faculty of Agriculture, Belgrade, Serbia
}

\begin{abstract}
Xanthomonas arboricola pv. corylina is the causal agent of bacterial blight of hazelnut. The bacterium has been listed as an A2 quarantine pathogen in Europe since 1978 and on the regulated non-quarantine pest list since 2019. Three isolates from various geographic regions and isolated at different times were sequenced using a hybrid approach with shortand long-read technologies to generate closed genome and plasmid sequences in order to better understand the biology of this pathogen.
\end{abstract}

\section{Genome Announcement}

Bacterial blight of hazelnut (Corylus spp.) was first reported in the early 20th century in Oregon (Barss 1913; Kałużna et al. 2021). The disease is caused by Xanthomonas arboricola pv. corylina and has since been reported in countries from all continents apart from Arctic and Antarctic (Kałużna et al. 2021). To limit the risk of introduction to other countries, especially via planting material, this Gram-negative bacterium was listed by the European Plant Protection Organization as an A2 quarantine pathogen in 1978 and as a regulated non-quarantine pest (Picard et al. 2018) since 2019 (European Union 2019).

The most important host for $X$. arboricola pv. corylina is Corylus avellana $\mathrm{L}$. (the common hazel) but other plant species such as Corylus pontica, Corylus maxima, and Corylus colurna were also found to be susceptible, although considered as minor hosts (OEPP/EPPO 1986, 2004).

Here, we report the complete genome sequences of three strains of $X$. arboricola pv. corylina (Table 1). The strains were isolated between 1939 and 2007 from either C. avellana or C. maxima and from three different countries (Table 1). These complete genomes should contribute to unveiling the ecology, evolution, and virulence of this economically relevant bacterium for hazelnut cultivation.

The CFBP $1159^{\mathrm{PT}}$ and CFBP 6600 strains were initially obtained as freeze-dried cultures in glass ampoules from the international strain collection Collection Française de Bactéries Associées aux Plantes (CFBP) (Beaucouzé, France). Strains were revived, stored, and handled as described previously (Dia et al. 2020). Strain Xac301 was isolated in 2007 in Poland from symptomatic leaf spots of a hazelnut (Puławska et al. 2010). Characteristic mucoid, yellow colonies were obtained and a pure colony initially called RIPF X12 (=Xac301) was further grown on yeast extract nutrient agar (YNA) medium. This isolate was identified as $X$. arboricola pv. corylina based on cellular fatty acid content converted to methyl esters as well as in gyrB

\footnotetext{
${ }^{\dagger}$ Corresponding author: J. F. Pothier; joel.pothier@zhaw.ch

J. F. Pothier and M. Kałużna contributed equally to this work.
}

The author(s) declare no conflict of interest.

Accepted for publication 10 October 2021.
Funding

A. Prokić was granted a Short-Term Scientific Mission by the European Cooperation in Science and Technology COST Action CA16107 EuroXanth to conduct some experiments in Wädenswil (Switzerland), and this work was partly financed by the National Science Centre, Poland (Narodowe Centrum Nauki), grant UMO- 2017/26/M/NZ9/ 01024 granted to M. Kałużna. Support was also provided to J. F. Pothier and F. Rezzonico by the Department of Life Sciences and Facility Management of the Zurich University of Applied Sciences (ZHAW) in Wädenswil; and to A. Obradovic by the Ministry of Education, Science and Technological Development, Republic of Serbia (Ministarstvo Prosvete, Nauke i Tehnološkog Razvoja) and the Faculty of Agriculture contract number 451-03-68/2020-14/200116.

\section{Keywords}

bacteria, bacterial pathogens, bioinformatics, complete genome, genomics, hazelnut, microbe-genome sequencing, Xanthomonas arboricola pv. corylina 
Table 1. Genome metrics and accession numbers of the newly sequenced Xanthomonas arboricola pv. corylina genomes

\begin{tabular}{|c|c|c|c|}
\hline \multirow[b]{2}{*}{ Variables $^{a}$} & \multicolumn{3}{|c|}{ Strain } \\
\hline & CFBP $1159^{\mathrm{PT}}$ & CFBP 6600 & Xac301 \\
\hline Origin (year) & United States (1939) & France (1977) & Poland (2007) \\
\hline Genome size (bp) & $5,080,866$ & $5,234,232$ & $5,294,219$ \\
\hline $\mathrm{G}+\mathrm{C}$ content $(\%)$ & 65.56 & 65.42 & 65.37 \\
\hline Total number of genes & 4,279 & 4,499 & 4,461 \\
\hline Number of plasmids & 0 & 1 & 1 \\
\hline Average read length (bp) & 301 & 301 & 251 \\
\hline Average coverage $(x)$ & 77 & 100 & 110 \\
\hline \multicolumn{4}{|l|}{ Oxford Nanopore data } \\
\hline Total number of reads & 50,946 & 27,923 & 129,031 \\
\hline Read length $\mathrm{N}_{50}(\mathrm{bp})$ & 32,779 & 38,810 & 22,574 \\
\hline Average coverage $(x)$ & 44 & 20 & 295 \\
\hline BUSCO score (\%) & 99.8 & 99.7 & 99.7 \\
\hline
\end{tabular}

gene fragment sequence analysis (Puławska et al. 2010). The gyrB sequence of Xac301 was most similar to the sequence of the $X$. arboricola pv. corylina pathotype strain (Fig. 1A). Koch's postulates were validated with strain Xac301 using leaf inoculation of hazelnut cultivars 'Webb's Prize Cob', 'Cosford', and 'Merveille de Bollwiller' (Fig. 1B). The isolate was stored in a $-80^{\circ} \mathrm{C}$ ultrafreezer in a mixture of $20 \%$ glycerol (vol/vol) and phosphate-buffered saline buffer $(0.27 \%$ $\mathrm{Na}_{2} \mathrm{HPO}_{4}, 0.04 \% \mathrm{NaH}_{2} \mathrm{PO}_{4}$, and $0.8 \% \mathrm{NaCl}$ ) until further use. Before extraction of DNA, Xac301 was revived and cultured on YNA medium and incubated at $26^{\circ} \mathrm{C}$ for 48 to $72 \mathrm{~h}$.

For strain Xac301, genomic DNA (gDNA) for both short- and long-read sequencing was isolated using the modified method of Aljanabi and Martinez (1997) from cells grown overnight at $26^{\circ} \mathrm{C}$ on YNA, as described previously (Kałużna et al. 2012). For short- read sequencing, library preparation was done using a NEBNext DNA Library Prep Master Mix Set for Illumina (NEB, Ipswich, MA, U.S.A.). Pooled libraries were sequenced on a MiSeq sequencer (Illumina, San Diego, CA, U.S.A.) with $2 \times 250$-bp paired-end reads using a MiSeq reagent kit, version 2 (Illumina).

For the CFBP $1159^{\mathrm{PT}}$ and CFBP 6600 strains, gDNA for Illumina MiSeq short-read sequencing was extracted from cells grown overnight at $28^{\circ} \mathrm{C}$ in nutrient yeast extract glycerol broth using the NucleoSpin tissue kit (Macherey-Nagel, Düren, Germany), according to the manufacturer's protocol. The quality of the gDNA was checked using a fragment analyzer (Advanced Analytical Technologies, Inc., Ankeny, IA, U.S.A.) and quantified using the Quant-iT PicoGreen double-stranded DNA quantification assay (Thermo Fisher Scientific, Waltham, MA, U.S.A.). Library preparation was done using the Nextera XT DNA library prep kit (Illumina) following the manufacturer's instructions. Sequencing of pooled libraries was performed on a MiSeq Illumina sequencer with $2 \times 300$-bp paired-end reads using a MiSeq reagent kit, version 3 (Illumina), according to the manufacturer's instructions.

For long-read sequencing, the gDNA of strains CFBP $1159^{\mathrm{PT}}$ and CFBP 6600 was extracted from overnight-grown cells using the Gentra PureGene Yeast/Bact kit protocol (Qiagen, Hilden, Germany). The gDNA quality was checked as described above and quantified using a high-sensitivity, double-stranded DNA quantitation kit (Allsheng, Hangzhou, China) and a Fluo-100B fluorometer (Allsheng).

For the three strains, long-read library preparation and sequencing were performed with the ligation sequencing kit (catalog number SQK-LSK109 for CFBP $1159^{\mathrm{PT}}$ and CFBP 6600 and catalog number SQK-LSK108 for Xac301; Oxford Nanopore Technologies, Oxford, United Kingdom) and run on an R9.4.1 flow cell with a MinION sequencer. The native barcoding 

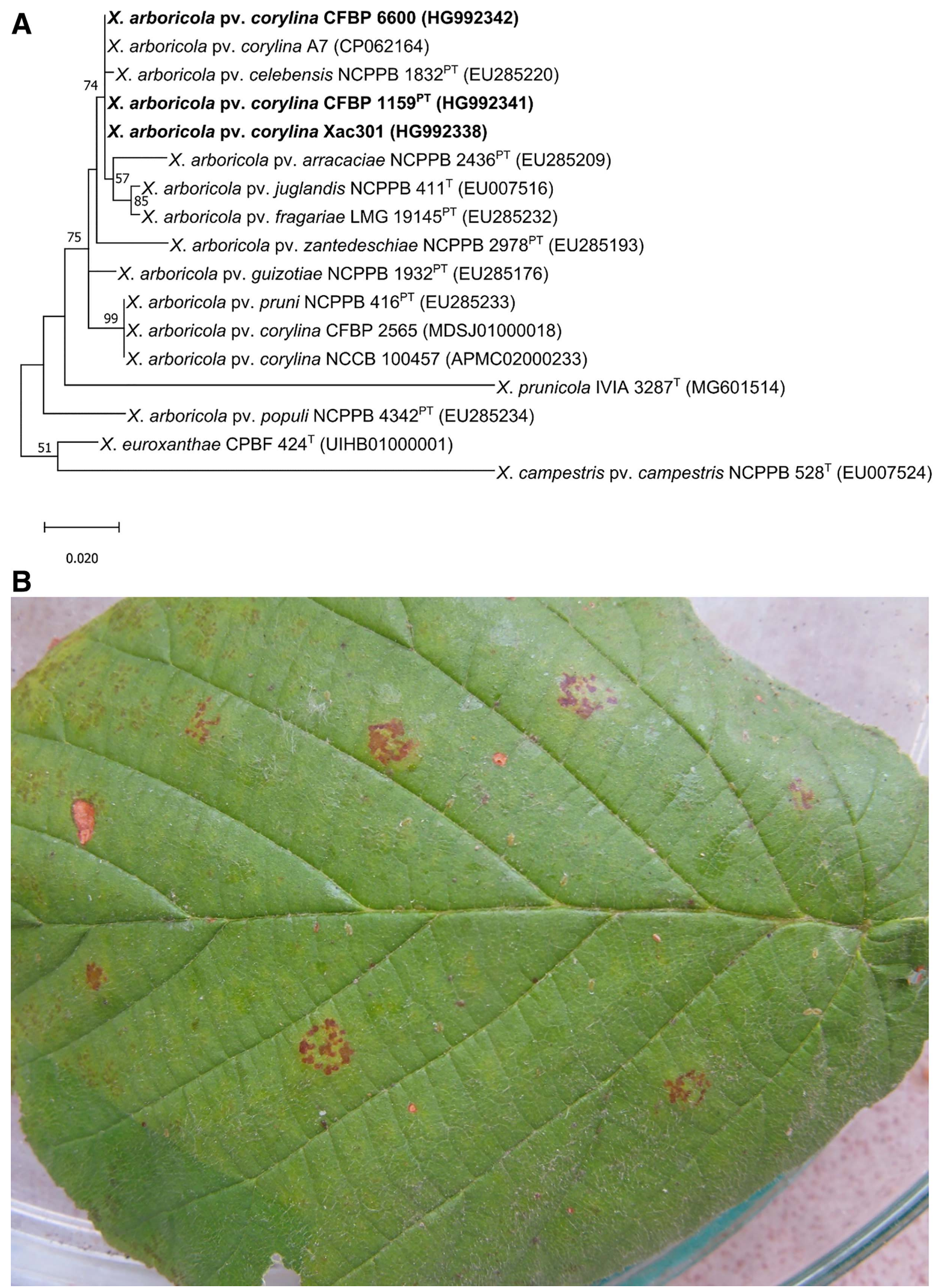

Fig. 1. A, Maximum-likelihood unrooted phylogenetic tree based on a 530-bp gyrB partial sequences of Xanthomonas strains. Phylogenetic and molecular evolutionary analyses were conducted using MEGA X, version 10.0.5 (Kumar et al. 2018). The alignment was obtained using the MUSCLE algorithm. The tree was constructed using the JTT matrix-based model. Percent bootstrap values calculated for 1,000 iterations are indicated near nodes and displayed only when $>50$. Accession numbers or source for gyrB sequences are indicated within parentheses next to the species name, with strains sequenced in this study marked in bold. Bar represents the expected number of substitutions per site. Superscripts following strain names: T indicates the type strain of a species and PT indicates the pathotype strain for a pathovar. B, Leaf spot symptoms developing on Corylus avellana 'Cosford' after syringe infiltration with Xanthomonas arboricola pv. corylina Xac301 and being kept for 10 weeks in the greenhouse under natural daylight conditions. Typical symptoms were already observed after 5 weeks postinoculation. 
Table 2. Comparison of the improved hybrid assembly versus the existing assembly of Xanthomonas arboricola pv. corylina CFBP $1159^{\mathrm{PT}}$

\begin{tabular}{lcc} 
Assembly name & GCA_905220785 & ASM293984v1 \\
Sequencing technology & Illumina MiSeq + Oxford Nanopore MinlON & Illumina HiSeq \\
Assembler & Trycycler v.0.3.3 + Pilon v.1.22 & Velvet v.1.2.07 + SOAPdenovo v.2.04 \\
Coverage & $121 \times$ & $100 \times$ \\
Total sequence length (bp) & $5,080,866$ & $5,105,973$ \\
Number of contigs & 1 & 124 \\
$\mathrm{~N}_{50}$ & $5,080,866$ & 135,548 \\
$\mathrm{G}+$ C content (\%) & 65.56 & 65.50 \\
Annotation pipeline & Prokka v.1.14.5 & NCBI PGAP v.4.2 \\
Number of & & 4,394 \\
$\quad$ Coding sequences & 4,214 & $1,1,1$ \\
$\quad$ Ribosomal RNAs (5S, 16S, 23S) & $2,2,2$ & 51 \\
$\quad$ Transfer RNAs & 57 & 99.8 \\
BUSCO score (\%) & 99.8 & \\
\hline
\end{tabular}

expansion kit (catalog number XP-NBD114) was used for multiplexing. Reads were basecalled and demultiplexed using Guppy, version 3.3.3.

Short- and long-read library preparation and sequencing were outsourced at Genomed S.A. (Warsaw, Poland) in the case of strain Xac301. For strains CFBP $1159^{\mathrm{PT}}$ and CFBP 6600 , these steps were outsourced at BSSE Genomics Facility (Basel, Switzerland) for shortread libraries and carried out in the Environmental Genomics and Systems Biology Research Group lab facilities (ZHAW) for long reads.

De novo hybrid assemblies using the MiSeq and MinION reads were conducted with Trycycler, version 0.3.3 (Wick et al. 2021). In total, 10,551, 10,533, and 199 nucleotide changes were performed during the first short-read polishing round for CFBP $1159^{\mathrm{PT}}$, CFBP 6600, and Xac301, respectively, using Pilon, version 1.22. The genomes were then annotated using Prokka, version 1.14.5 (Seemann 2014). All tools were run with default parameters unless otherwise specified.

The size of the hybrid assemblies ranged from $5,080,866$ to $5,294,219 \mathrm{bp}$, a size range typically found in Xanthomonas genomes (Table 1). The $\mathrm{G}+\mathrm{C}$ contents of the genomes varied from 65.37 to $65.56 \%$, also comparable with other Xanthomonas spp. $\mathrm{G}+\mathrm{C}$ contents. Wholegenome comparison based on average nucleotide identity using BLASTN (ANIb) implemented in pyANI, version 0.2.10 (Pritchard et al. 2016), confirmed that the three strains had high degree of synteny between them (Table 1) and to other $X$. arboricola genomes (data not shown). Genome completeness varied between 99.7 and $99.8 \%$ (Table 1) when assessed using the Benchmarking Universal Single-Copy Orthologs (BUSCO), version 5.2.1 (Manni et al. 2021) and the xanthomonodales_odb10 (2020-03-06) lineage dataset.

Because an assembly already existed for the pathotype strain CFBP $1159^{\mathrm{PT}}$, a comparison was performed versus the hybrid assembly presented here which revealed some improvements and few minor differences (Table 2).

A single 24-kb plasmid was present in the final assemblies of CFBP 6600 and Xac301. This plasmid contains the type three effector (T3E) XopAG (HopG1) and the avirulence protein XopE2. The presence of XopAG in two of the three strains and the plasmid-borne localization of this T3E agrees with previous observations from a draft genome sequence of this same pathovar (Ibarra Caballero et al. 2013). In the genome of CFBP $1159^{\mathrm{PT}}$, XopE2 was detected on the chromosome. The T3E AvrBs3 was also detected in the genome of the CFBP $1159^{\mathrm{PT}}$ pathotype strain whereas it is absent from the two other genomes presented in this work, as previously reported from another draft genome of this same pathovar (Ibarra Caballero et al. 2013). The presence of the $\operatorname{cop} A B$ operon and $\operatorname{cop} L$ gene, whose products are involved in copper resistance in this pathogenic bacteria (Kałużna et al. 2021), was detected in the chromosome of all three strains. Similarly, cutC and pCuAC genes, whose product could be implicated in the survival of this bacterium at high copper concentration (Nuñez Cerda et al. 2022), were also found in all three strains.

The sequenced genomes discussed here will be used for further analysis of evolution within the species $X$. arboricola, producing a better understanding of the pathogenicity and virulence as well as development of improved tools for diagnostics of this relevant pathogen for the worldwide production of hazelnut. 


\section{Data Availability}

The raw data and assembled and annotated genome sequences have been deposited in the European Nucleotide Archive under BioProject number PRJEB42844. The genome and raw read accession numbers for each strain are shown in Table 1.

\section{Acknowledgments}

We thank the HPC team of the School for Life Sciences and Facility Management at ZHAW for computer resources and support. This article is based upon work from COST Action CA16107 EuroXanth, supported by COST (European Cooperation in Science and Technology).

\section{Literature Cited}

Aljanabi, S. M., and Martinez, I. 1997. Universal and rapid salt-extraction of high quality genomic DNA for PCR-based techniques. Nucleic Acids Res. 25:4692-4693.

Barss, H. P. 1913. A new filbert disease in Oregon. Oreg. Agric. Exp. Stn. Bienn. Crop Pest Hortic. Rep. 14:213-223.

Dia, N. C., Rezzonico, F., Smits, T. H. M., and Pothier, J. F. 2020. Complete or highquality draft genome sequences of six Xanthomonas hortorum strains sequenced with short- and long-read technologies. Microbiol. Resour. Announce. 9:e00828-20.

European Union. 2019. Commission implementing directive (EU) 2019/2072 of 28 November 2019 establishing uniform conditions for the implementation of regulation (EU) 2016/2031 of the European Parliament and the Council, as regards protective measures against pests of plants, and repealing commission regulation (EC) no. 690/2008 and amending commission implementing regulation (EU) 2018/2019. Off. J. Eur. Union L319:1-278.

Ibarra Caballero, J., Zerillo, M. M., Snelling, J., Boucher, C., and Tisserat, N. 2013. Genome sequence of Xanthomonas arboricola pv. corylina, isolated from Turkish filbert in Colorado. Genome Announce. 1:e00246-13.

Kałużna, M., Fischer-Le Saux, M., Pothier, J. F., Jacques, M.-A., Obradović, A., Tavares, F., and Stefani, E. 2021. Xanthomonas arboricola pv. juglandis and pv. corylina: Brothers or distant relatives? Genetic clues, epidemiology, and insights for disease management. Mol. Plant Pathol. 22:1481-1499.

Kałużna, M., Janse, J. D., and Young, J. M. 2012. Detection and identification methods and new tests as used and developed in the framework of COST 873 for bacteria pathogenic to stone fruits and nuts: Pseudomonas syringae pathovars. J. Plant Pathol. 94:S1.117-S1.126.

Kumar, S., Stecher, G., Li, M., Knyaz, C., and Tamura, K. 2018. MEGA X: Molecular evolutionary genetics analysis across computing platforms. Mol. Biol. Evol. 35 : 1547-1549.
Manni, M., Berkeley, M. R., Seppey, M., Simão, F. A., and Zdobnov, E. M. 2021. BUSCO update: Novel and streamlined workflows along with broader and deeper phylogenetic coverage for scoring of eukaryotic, prokaryotic, and viral genomes. Mol. Biol. Evol. 38:4647-4654.

Nuñez Cerda, P. D., Muster, C., Lisperguer, M. J., Vargas, E., and Bustos, S. 2022. Complete genome of Xanthomonas arboricola pv. corylina strain A7 isolated from southern Chile. Mol. Plant-Microbe Interact. 35:94-95.

OEPP/EPPO. 1986. Data sheets on quarantine organisms No. 134, Xanthomonas campestris pv. corylina. Bull. OEPP/EPPO Bull. 16:13-16.

OEPP/EPPO. 2004. Xanthomonas arboricola pv. corylina. Bull. OEPP/EPPO Bull. 34:179-181.

Picard, C., Afonso, T., Benko-Beloglavec, A., Karadjova, O., Matthews-Berry, S., Paunovic, S. A., Pietsch, M., Reed, P., van der Gaag, D. J., and Ward, M. 2018. Recommended regulated non-quarantine pests (RNQPs), associated thresholds and risk management measures in the European and Mediterranean region. Bull. OEPP/EPPO Bull. 48:552-568.

Pritchard, L., Glover, R. H., Humphris, S., Elphinstone, J. G., and Toth, I. K. 2016. Genomics and taxonomy in diagnostics for food security: Soft-rotting enterobacterial plant pathogens. Anal. Methods 8:12-24.

Puławska, J., Kałużna, M., Kołodziejska, A., and Sobiczewski, P. 2010. Identification and characterization of Xanthomonas arboricola pv. corylina causing bacterial blight of hazelnut: A new disease in Poland. J. Plant Pathol. 92:803-806.

Seemann, T. 2014. Prokka: Rapid prokaryotic genome annotation. Bioinformatics 30: 2068-2069.

Wick, R. R., Judd, L. M., Cerdeira, L. T., Hawkey, J., Méric, G., Vezina, B., Wyres, K. L., and Holt, K. E. 2021. Trycycler: Consensus long-read assemblies for bacterial genomes. bioRxiv. https://www.biorxiv.org/content/10.1101/2021.07.04. $451066 \mathrm{v} 1$ 